Methods A total of 228 acute coronary syndrome (ACS) patients were randomly divided into standard statin group (SSG, $n=115)$ and intensive statin group (ISG, $\mathrm{n}=113$ ). Patients in SSG received $20 \mathrm{mg}$ simvastatin and patients in ISG received $80 \mathrm{mg}$ simvastatin for 7 days before PCI. TIMI grade flow (TGF), corrected TIMI frame count (CTFC) and TIMI myocardial perfusion grade (TMPG) of the intervened vessel were recorded before and after stent deployment. Plasma level of CK-MB and cTnI were measured before and $24 \mathrm{~h}$ after the procedure.

Results The TFG after stent deployment was significantly improved with less TIMI $0-1$ patients and more TIMI 3 blood flow in ISG than in SSG (all $\mathrm{p}<0.05)$. Patients with no reflow phenomenon were less in ISG $(p<0.001)$. The CTFC was lower in ISG than SSG $(p<0.001)$. TMPG was also improved in ISG than SSG $(p=0.001)$. $24 \mathrm{~h}$ after the procedure, although PCI caused significantly increase in CK-MB, the elevated CK-MB value was lower in ISG than SSG (18.74 \pm 8.41 vs $21.78 \pm 10.64 \mathrm{p}=0.018)$. Similar changes were also found with regard to Troponin I ( $0.99 \pm 1.07$ vs $1.47 \pm 1.54, p=0.006)$. No myocardial infarction was found. Among them, myocardial necrosis was detected in 13\% of the patients in SSG, while $4.4 \%$ in ISG $(p=0.021)$. Myocardial infarction was found in $4.4 \%$ in the patients in SSG and $0.9 \%$ in ISG $(p=0.213)$.

Conclusion Intensive statin pretreatment for 7 days before PCI can further improve myocardial blood perfusion, protect myocardium from ischaemic injury.

\section{e0525 PROTECTIVE EFFECT OF SIMVASTATIN COMBINED WITH ANISODAMINE ON MYOCARDIAL PERFUSION IN SWINE NO REFLOW MODEL}

doi:10.1136/hrt.2010.208967.525

Fu Xianghua, Jia Xinwei, Wang Yanbo, Wang Xuechao, Zhang Jing, Fan Weize, Hao Guozhen. The second Hospital of Hebei Medical University, Shijiazhuang, Hebei, China

Objectives To evaluated the preventive effect of simvastatin combined with anisodamine on myocardial perfusion in no reflow, and to probe the possible mechanism.

Method Totally 16 minipig of $30-40 \mathrm{Kg}$ were randomly divided into anisodamine groups $(A, n=8)$ and anisodamine plus simvastatin group $(A+S, n=8)$. Pigs in Group $A+S$ were pretreated with oral simvastatin for 7 days, while pigs in A groups were given placebo. Seven days later, CAG was performed, and the dopper wire was used to record blood velocity. The pressure of aorta ( $\mathrm{Pa})$ was monitored. PMBS was injected to establish no reflow model. Anisodamine was injected into the LAD 2 min before PBMS was injected. The TIMI blood flow, TMPG and CTFC were recorded to evaluate the myocardial perfusion. The sample of myocardium in ischaemic zone and normal zone were measured. Blood sample was taken before and after the experiment to measure the level of CK-MB, cTnI and hs-CRP. The percent of necrotic myocardium was calculated by myocardium stain method.

Results The TIMI blood flow and TFCs were better in Group A+S $(p<0.05)$. The Pa was increased in both groups after PMBS injection at the early stage $(p<0.01)$, and then it begun to decrease in Group A $(p<0.05)$, while it remained its high level in Group $A+S(p=0.042)$. The bAPV was increased in both groups, which was more obvious in the Group A after PMBS injection. After the injection of PMBS, the hAPV was significantly decreased in both groups $(p<0.01)$, but it was still higher in group $A+S(p=0.000)$. The CFR was continuously deceased after the PMBS injection $(p<0.05)$, but it was higher in Group $A+S(p=0.025)$. The $h-M R$ was further increased $(p=0.024)$, with no difference between two groups after the PMBS injection. The level of serum cholesterol was similar between the two groups $(p=0.063)$. CK-MB, TnI, hs-CRP and MDA were increased after the experiment, with the higher levels in Group A $\mathrm{NO}$ was also increased $(p=0.000)$, with the higher level in Group $A+S(p=0.006)$. SOD was decreased $(p=0.000)$ in both groups, with lower level in Group A ( $p=0.000)$. The infarcted size in group A was larger than that in $A+S$ group $(p<0.05)$.

Conclusion Simvastatin combined with anisodamine can significantly improve myocardial blood perfusion and protect the myocardium against ischaemic injury during PCI. The possible mechanism involves improving of coronary haemodynamics, antiinflammation and antioxidation.

\section{e0526 PROTECTIVE EFFECTS OF INTENSIVE STATIN PRETREATMENT ON RENAL FUNCTION IN PATIENTS WITH ACUTE CORONARY SYNDROME UNDERGOING PERCUTANEOUS INTERVENTION}

doi:10.1136/hrt.2010.208967.526

Fu Xianghua, Jia Xinwei, Wang Yanbo, Wang Xuechao, Zhang Jing, Fan Weize, Hao Guozhen, Jiang Yunfa. The Second Hospital of Hebei Medical University, Shijiazhuang, Hebei, China

Objectives To evaluate the protective effects of higher dose statin on renal function and the incidence of CIN.

Methods 228 patients with acute coronary syndrome undergoing delayed percutaneous coronary intervention were randomly divided into standard statin group (SSG $\mathrm{n}=115$ ) and intensive statin group (ISG $\mathrm{n}=113$ ). Patients in SSG were given simvastatin $20 \mathrm{mg} /$ day and patients in ISG were given simvastatin $80 \mathrm{mg} /$ day for at least 7 days before PCI, Serum creatinine was measured at admission, $24 \mathrm{~h}$ and $48 \mathrm{~h}$ after PCI, and the Creatinine clearance was calculated. The levels of hs-CRP, ICAM-1 and P-selectin were also measured.

Results Serum creatinine underwent significant increase after PCI, the peak value occurred at $24 \mathrm{~h}$, and then began to decrease. At $48 \mathrm{~h}$ after PCI, the creatinine level significantly decreased $(p<0.001)$ to baseline level in ISG, whereas in SSG the creatinine level failed to decrease significantly. Serum creatinine at admission was not significantly different between the two groups, But at 24th and 48th hour after PCI, it were lower in ISG than SSG $(p<0.05$ at 24 th hour and $p<0.001$ at 48 th hour). The creatinine clearance significantly decreased after PCI, the lowest value occurred at $24 \mathrm{~h}$, and then it began to increase. In SSG, the creatinine clearance increased significantly $(p=0.03)$ at $48 \mathrm{~h}$, but still significantly lower than baseline level. In ISG, the creatinine clearance increased significantly $(\mathrm{p}<0.001)$ at $48 \mathrm{~h}$ and recovered to the level at baseline. Creatinine clearance improved much more in ISG at 24 and $48 \mathrm{~h}$ than that in SSG $(p<0.001$ at 24 th hour and at 48 th hour). Although procedure caused significant increase in hs-CRP, P-selectin and ICAM-1 $(p<0.001)$, the increase in ISG was smaller than SSG $(p<0.001)$.

Conclusion Pretreatment with intensive statin dosage before PCI can further decrease the occurrence of CIN. This benefit may be associated with the lowering of hs-CRP, P-selectin and ICAM levels.

\section{e0527 INTRAVASCULAR ULTRASOUND CRITERIA FOR THE ASSESSMENT OF THE FUNCTIONAL SIGNIFICANCE OF INTERMEDIATE CORONARY ARTERY STENOSIS}

doi:10.1136/hrt.2010.208967.527

Cheng Xunmin, Jiang Shisen. Cardiology Department, Nanjing General Hospital of Nangjing Millitary Command of Pla, Nangjing

Introduction In recent years, intravascular ultrasound (IVUS) has evolved as a valuable adjunct to angiography. IVUS allows precise tomographic measurement of lumen area and plaque size, distribution and, to some extent, composition. It is essential in clinic decision 